Special Issue of the 7th International Advances in Applied Physics and Materials Science (APMAS 2017)

\title{
Reversibility Algorithm for 2D Cellular Automata with Reflective Condition
}

\author{
S. REDJEPOV ${ }^{a}$, E. ACAR ${ }^{b, *}$ AND S. UGUZ ${ }^{c}$ \\ ${ }^{a}$ Faculty of Computer Engineering, Tashkent University of Information Technologies, Tashkent, 100202, Uzbekistan \\ ${ }^{b}$ Department of Mathematics, Harran University, Sanliurfa, 63120, Turkey \\ ${ }^{c}$ SU Mathematics and Science Academy, S. Urfa, 63100, Turkey
}

\begin{abstract}
In the present paper, there are studied main theoretical views of two-dimensional (2D) linear uniform cellular automata with reflective boundary condition over the ternary field, i.e. three states spin case or $\mathrm{Z}_{3}$. We set up a relation between reversibility of cellular automata and characterization of 2D uniform linear cellular automata with this special boundary conditions by using of the matrix theory. In near future, these cellular automata can be found in many different real life applications, e.g. computability theory, theoretical biology, image processing area, textile design, video processing, etc.
\end{abstract}

DOI: 10.12693/APhysPolA.134.454

PACS/topics: reversibility, 2D CA, rule matrix, reflective boundary, ternary field

\section{Introduction}

A cellular automaton (cellular automata $(\mathrm{CA})$ for plural) is a discrete dynamical model. CA operate infinite space and time and their behaviors are entirely characterized by defined special some local given rules. Up to now researchers investigated and explored many aspects of CA with special interest in the study of different kinds of CA. These studies are mainly some special analytical ideas into the behavior of the iterative process. CA idea are firstly investigated by Von Neumann and Burks [1] around 1950's, were in systematic way investigated by Hedlund considering its just mathematics theoretical view. Von Neumann was interested in the association between biology and the new study of CA. Onedimensional (1D) CA are studied in very extended mathematical ways and demonstrated for many various applications. However, not so much interest is presented to 2D uniform and hybrid CA. Von Neumann presented that a cellular automaton can be more universal considering its many elegant properties. Due to its structured complexness, Von Neumann rules never proceeded in computer program language codes in that time. Around the eighties, Wolfram [2] has started to study some prominent properties of a simple 1D uniform CA rules and investigated that even different simple rules are capable of showing very interesting complex and different chaotic behaviors. Uniform linear CA have been admitted striking interest in the past decades [3-11]. The papers $\left[4,8^{-}\right.$ 12] investigate the mathematical analytic behavior of the hybrid and uniform $2 \mathrm{D} \mathrm{CA}$ with states values in ternary fields $Z_{3}$. In this paper, we concentrate a special family of $2 \mathrm{D}$ finite linear $\mathrm{CA}$ with reflective condition over

*corresponding author; e-mail: karakusecem@harran.edu.tr the field $Z_{3}$. Here, we set up a specific relation between reversibility of uniform cellular automata and characterization of 2D CA with reflective boundary condition. We study the determination of the characterization problem of this special CA by means of the matrix algebra theory. Using the linear rule matrices presented in the work, the results give further to the algebraic consequences of these 2D CA and relates some real-life applications found by the authors in the literature (i.e. [8, 9-19]).

\section{Reversibility of linear CA (rule $2460 \mathrm{RB}$ )}

We work on CA established by uniform linear rule over the three states field $\mathrm{Z}_{3}$ and deal with the general case of the CA reversibility problem. The determining all $1 \mathrm{D}-2 \mathrm{D}$ uniform CA is reversible or not is generally a challenging problem. Firstly, there are found the rule matrices $\mathrm{T}_{\text {rules }}$ corresponding to finite 2D linear $\mathrm{CA}$, after then there is characterized the reversibility problem of this special reflexive $2 \mathrm{D}$ CA.

\subsection{Rule matrices of linear $C A$}

Considering the neighbourhood of the information cells, there are following known studied boundary approaches in the literature.

- Null boundary (NB): the boundary cells are zero spin values, or fixed 0-state.

- Periodic boundary (PB): the boundary cells are contiguous to each border way.

- Reflective boundary (RB): the boundary cells are reflection to border way.

An important note is that there are also different types of special boundary conditions to study for future studies, i.e. adiabatic boundary, etc. (see details $[10,15-19]$ ). 
Transition rule matrices for reflexive case: Rule matrix under reflective boundary for rule $2460 \mathrm{RB}$ :

$$
T_{2460 R B}=
$$

$$
\left(\begin{array}{cccccc}
a I+R(b, d) & c I & 0 & \cdots & 0 & 0 \\
a I & R(b, d) & c I & \cdots & 0 & 0 \\
0 & a I & R(b, d) & \cdots & 0 & 0 \\
\vdots & \vdots & \vdots & \vdots & \vdots & \vdots \\
\vdots & \vdots & \vdots & \vdots & \vdots & \vdots \\
0 & 0 & 0 & \cdots & a I & c I+R(b, d)
\end{array}\right)
$$

where each partioned matrix are

$$
R(b, d)=\left(\begin{array}{lll}
d & b & 0 \\
d & 0 & b \\
0 & d & b
\end{array}\right)
$$

Lemma: For uniform type $\mathrm{CA}$ over $\mathrm{Z}_{3}$, the representation of the fundamental linear CA rule matrices $\left(3^{0}=1\right.$; $3^{1}=3 ; 3^{2}=9 ; 3^{3}=27 ; 3^{4}=81 ; 3^{5}=243 ; 3^{6}=729$; $3^{7}=2187$ and $3^{8}=6561$ ) with reflective boundary neighbourhood over $\mathrm{Z}_{3}$ is found by considering the fixed block matrix $\mathrm{T}_{2460 R B}$ defined above.

\subsection{Reversibility idea of $2 D$ uniform linear $C A$}

If the transition rule matrix $T_{\text {rules }}$ is a non-singular matrix, then we have $X(t)=\left(T_{\text {rules }}\right)^{-1} X^{(t+1)}(\bmod 3)$. Here a main problem of finding whether the rule matrix $T_{\text {rules }}$ is invertible (or nonsingular) matrix or not for any cases. If the transition matrices $T_{\text {rules }}$ have full rank properties, then it is said to be a invertible CA, hence the $2 \mathrm{D}$ finite hybrid or uniform CA is a reversible one, otherwise it is called a irreversible CA. The analysis on reversible or irreversible cases of the transition rules found in the next subsection is given as a Theorem.

\section{Rule block matrices of CA with reflective boundary}

Let $T_{i}$ indicate the $i$-th row entry and $T_{i}[j]$ indicate the $j$-th position element of the $i$-th row of rule matrix $T_{\text {rules }}$, repectively. We set up a specific relation between reversibility of uniform $\mathrm{CA}$ and characterization of $2 \mathrm{D}$ CA with reflective boundary condition by means of the matrix algebra.

Main theorem: Let the matrix

$$
\left(T_{\text {rules }}\right)_{m n \times m n}=
$$

$$
\left(\begin{array}{cccccc}
a I+R(b, d) & c I & 0 & \cdots & 0 & 0 \\
a I & R(b, d) & c I & \cdots & 0 & 0 \\
0 & a I & R(b, d) & \cdots & 0 & 0 \\
\vdots & \vdots & \vdots & \vdots & \vdots & \vdots \\
\vdots & \vdots & \vdots & \vdots & \vdots & \vdots \\
0 & 0 & 0 & \cdots & a I & c I+R(b, d)
\end{array}\right)
$$

with $m, n \geq 3$ representing the linear $2 \mathrm{D} \mathrm{CA}$ over the field $\mathrm{Z}_{3}$ under reflective boundary condition. Let

$$
T_{1}^{1}=T_{1}, \quad T_{1}^{k+1}=-T_{1}^{k}[k]\left(T_{k+1}^{1}[k]\right)^{-1} T_{k+1}^{1}+T_{1}^{k} .
$$

Define the following $1 \times 1$ block matrix consisting of blocks of square matrices of order $n K=\left(T_{1}^{m}[m]\right)$. Hence we obtain that the rank equalites of the transtition matrix is presented by

$$
\operatorname{rank}\left(T_{\text {rules }}\right)=(m-1) n+\operatorname{rank}(K) .
$$

Proof: Let use induction on $m$ and utilize the idea similarly used in the calculation of the rank of Trules, then we will find the linear algebraic relation of the rank of Trules for any cases.

$$
\begin{gathered}
T_{2460 R B}= \\
\left(\begin{array}{cccccc}
a I+R(b, d) & c I & 0 & \cdots & 0 & 0 \\
a I & R(b, d) & c I & \cdots & 0 & 0 \\
0 & a I & R(b, d) & \cdots & 0 & 0 \\
\vdots & \vdots & \vdots & \vdots & \vdots & \vdots \\
\vdots & \vdots & \vdots & \vdots & \vdots & \vdots \\
0 & 0 & 0 & \cdots & a I & c I+R(b, d)
\end{array}\right)
\end{gathered}
$$

Firstly, one can see above that the sub-matrix comprising of all rows except the first is the upper triangular shape and sub-matrix is of a full rank matrix that the sub-matrix rank is $(m-1) n$ for $a, b, c, d, e \in Z_{3}^{*}=$ $\{1,2\}$. Now, if one can multiply the second row $T_{2}$ by $-T_{1}^{1}[1] .\left(T_{2}^{1}[1]\right)^{-1} T_{2}^{1}$ and adding that product to $T_{1}^{1}$, so first entry elements of the first new-one row $T_{1}^{2}$ becomes zero. Hence, we change the the first row by $T_{1}^{2}=-T_{1}^{1}[1] \cdot\left(T_{2}^{1}[1]\right)^{-1} T_{2}^{1}+T_{1}^{1}$. Now, we want to $T_{1}^{2}[2] \mathrm{s}$ zero. In that case, we apply the same thing for $T_{1}^{2}$. So we have $T_{1}^{3}=-T_{1}^{2}[2] .\left(T_{3}^{1}[2]\right)^{-1} T_{3}^{1}+T_{1}^{2}$. Thus the second entry-elements of the new first-row becomes zero. Repeatedly, we see that subsequently $m-1$ steps the only non-zero entires of $T_{1}^{m-1}$ is $T_{1}^{m}[m]$.

$T_{2460 R B}=$

$$
\left(\begin{array}{cccccc}
0 & 0 & 0 & \cdots & 0 & T_{1}^{m}[m] \\
a I & R(b, d) & c I & \cdots & 0 & 0 \\
0 & a I & R(b, d) & \cdots & 0 & 0 \\
\vdots & \vdots & \vdots & \vdots & \vdots & \vdots \\
\vdots & \vdots & \vdots & \vdots & \vdots & \vdots \\
0 & 0 & 0 & \cdots & a I & c I+R(b, d)
\end{array}\right)=\left(\begin{array}{cc}
0 & K \\
L & M
\end{array}\right)
$$

The left lower block of L has full rank $(m-1) . n$. Hence we can obtain that the rank equalites of the transtition matrix is presented by

$$
\operatorname{rank}\left(T_{\text {rules }}\right)=\operatorname{rank}(L)+\operatorname{rank}(K)=(m-1) n+\operatorname{rank}(K) .
$$

3.1. Image problems and reflexive rule $2460 C A$

Self-copied pattern producing is one of the most interesting topic and research area in nonlinear science. 


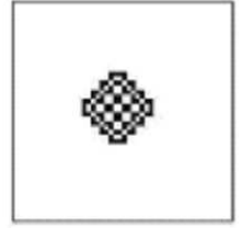

First image

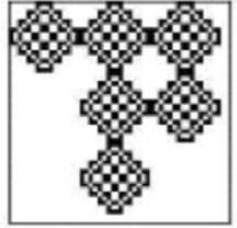

Rule 2460 NB

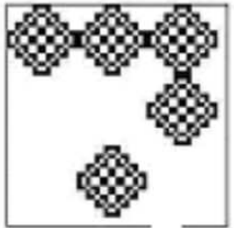

Rule 2460 RB
Fig. 1. Image applications of null (NB) and reflective (RB) boundary CA rule $2460 \mathrm{NB}-\mathrm{RB}$ for $2 \mathrm{D}$.

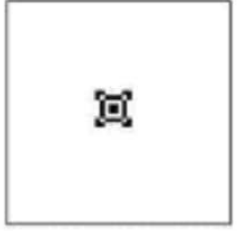

First image

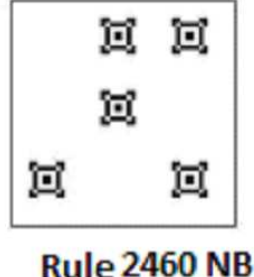

Rule 2460 NB

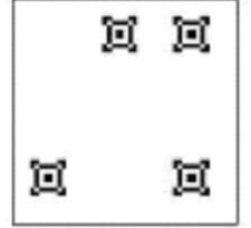

Rule 2460 RB
Fig. 2. Image applications of null (NB) and reflective (RB) boundary CA rule 2460 NB-RB for 1D.

Pattern generation is the process of transforming copies of the motif about the array (1D), plane (2D) or space (3D) in order to create the whole repeating pattern with no overlaps and blank (Figs. 1, 2) [5, 6, 9-13]. These patterns have some mathematical properties which make generating algorithm possible. $\mathrm{CA}$ is a good candidate algorithmic approach used for pattern generation. We obtain the following pattern generation of $t=16$ iteration of rule 2460 NB-RB (Figs. 1, 2). Self-copied patterns given in Figs. 1, 2 can be generated only when number of repetition is 16 iterations. Also, behaviors for different boundaries produce different shapes when $t=16$. These interesting results should be investigated as image problem for $\mathrm{CA}$ in the next studies.

\section{Conclusions}

We study the theoretical aspects of 2D linear CA with reflective boundary case over the ternary field $\mathrm{Z}_{3}$ or three spin states case. It is introduced a main theorem for determining the reversibility of these CA for a general case of linear transformation. Also after constructing the transition matrix representation of $2 \mathrm{D}$ linear $\mathrm{CA}$, we have found image applications (see Figs. 1, 2) for the 2D linear CA. New other results is another goal of the future study. These CA results can be applied successfully in especially image processing area [9-13] and the other science branches in near future [14-21].

\section{References}

[1] J. Von Neumann, A.W. Burks, The Theory of SelfReproducing Automata, University of Illinois Press, Urbana 1966.

[2] S. Wolfram, Rev. Mod. Phys. 55, 601 (1983).

[3] H. Akın, I. Siap, S. Uguz, AIP Conf. Proc. 1309, 16 (2010).

[4] P. Chattopadhyay, P.P. Choudhury, K. Dihidar, Comp. Math. Appl. 38, 207 (1999).

[5] P.P. Choudhury, S. Sahoo, S.S. Hassan, S. Basu, D. Ghosh, D. Kar, Ab. Ghosh, Av. Ghosh, A.K. Ghosh, Int. J. Comp. Cogn. 8, 50 (2010).

[6] H.H. Chou, J.A. Reggia, Physica D 110, 252 (1997).

[7] K. Dihidar, P.P. Choudhury, Inf. Sci. 165, 91 (2004).

[8] J. Gravner, G. Gliner, M. Pelfrey, Physica D 240, 1460 (2011)

[9] U. Sahin, F. Sahin, S. Uguz, in: Proc. 10th IEEE Conf. on High Capacity Optical Networks and Emerging/Enabling Technologies, Magosa (Cyprus), 2013, p. 228 .

[10] U. Sahin, S. Uguz, H. Akin, Int. J. Bifurc. Chaos 25, 1550011 (2015).

[11] U. Sahin, S. Uguz, H. Akin, I. Siap, Appl. Math. Model. 39, 2003 (2015).

[12] U. Sahin, S. Uguz, F. Sahin, Comp. Elect. Eng. 40 , 59 (2014)

[13] I. Siap, H. Akın, S. Uguz, Comp. Math. Appl. 62 4161 (2011).

[14] S. Uguz, H. Akın, I. Siap, Int. J. Bifurc. Chaos 23 , 1350101 (2013).

[15] S. Uguz, U. Sahin, H. Akın, I. Siap, Int. J. Bifurc. Chaos 24, 1430002 (2014).

[16] S. Uguz, U. Sahin, H. Akın, I. Siap, Acta Phys. Pol. A 125, 435 (2014).

[17] S. Uguz, U. Sahin, F. Sahin, Comp. Electr. Eng. 43, 180 (2015).

[18] S. Uguz, H. Akin, I. Siap, U. Sahin, Appl. Math. Model. 40, 8017 (2016).

[19] S. Uguz, I. Siap, H. Akın, Acta Phys. Pol. A 123, 480 (2013).

[20] E. Acar, S. Uguz, H. Akın, Acta Phys. Pol. A 131 , 432 (2017).

[21] S. Uguz, S. Redjepov, E. Acar, H. Akın, Int. J. Bifurc. Chaos 27, 1750083 (2017). 